\title{
Artifact Reduction Based on Sinogram Interpolation for the 3D Reconstruction of Nanoparticles Using Electron Tomography
}

\author{
Kadir Sentosun, Ivan Lobato, Eva Bladt, Yang Zhang, Willem Jan Palenstijn, \\ Kees Joost Batenburg, Dirk Van Dyck, and Sara Bals*
}

Electron tomography is a well-known technique providing a 3D characterization of the morphology and chemical composition of nanoparticles. However, several reasons hamper the acquisition of tilt series with a large number of projection images, which deteriorate the quality of the $3 \mathrm{D}$ reconstruction. Here, an inpainting method that is based on sinogram interpolation is proposed, which enables one to reduce artifacts in the reconstruction related to a limited tilt series of projection images. The advantages of the approach will be demonstrated for the 3D characterization of nanoparticles using phantoms and several case studies.

\section{Introduction}

Transmission electron microscopy (TEM) is a valuable technique to investigate nanomaterials yielding both structural and chemical information at the atomic scale. However, images acquired by TEM are typically $2 \mathrm{D}$ projection images of 3D objects. Therefore, it is not straightforward to investigate aspects that are intrinsically related to the $3 \mathrm{D}$ structure such as the surface to volume ratio or the surface facets of nanomaterials. However, precise knowledge of these characteristics is required to understand, e.g., the catalytic and optical properties of nanostructures. ${ }^{[1-5]}$ For such applications, an accurate reconstruction of the shape is often more important than details concerning the internal structure.

K. Sentosun, Dr. I. Lobato, Dr. E. Bladt, Dr. Y. Zhang, Prof. D. Van Dyck Prof. S. Bals

EMAT-University of Antwerp

Groenenborgerlaan 171, B-2020 Antwerp, Belgium

E-mail: Sara.bals@uantwerpen.be

Dr. W. J. Palenstijn, Prof. K. J. Batenburg

Centrum Wiskunde and Informatica

Scientific Computing Group

Science Park 123, NL-1098XG Amsterdam, The Netherlands

Prof. K. J. Batenburg

Wiskunde en Natuurwetenschappen

Mathematical Institute

Leiden University

Niels Bohrweg 1, 2333 CA Leiden, The Netherlands

The ORCID identification number(s) for the author(s) of this article can be found under https://doi.org/10.1002/ppsc.201700287.

DOI: $10.1002 / p p s c .201700287$
3D electron microscopy or "electron tomography" is a technique based on the acquisition of a tilt series of $2 \mathrm{D}$ projection images at different tilt angles, which is used as an input for a mathematical algorithm yielding a $3 \mathrm{D}$ reconstruction of the original object. ${ }^{[6,7]}$ In materials science, projection images are mostly acquired using high angle annular dark field (HAADF) scanning transmission electron microscopy (STEM). ${ }^{[1-3]}$ The method has already been applied to a large variety of materials such as (supported) nanoparticles, ${ }^{[3]}$ assemblies of nanoparticles, ${ }^{[8]}$ and porous materials. ${ }^{[9]}$ Recently, electron tomography was furthermore combined with analytical techniques such as X-ray energy-dispersive spectroscopy ${ }^{[10,11]}$ and electron energy loss spectroscopy ${ }^{[12,13]}$ yielding both structural and chemical information.

Typically, a tilt series of projection images is acquired over a tilt range of $\pm 70^{\circ}-80^{\circ}$ with a tilt increment of $2^{\circ}$. Unfortunately, for beam sensitive materials, it is far from straightforward to acquire projection data every $2^{\circ}$ since the electron beam may induce changes in the morphology or even sample damage during the acquisition. To avoid this, the number of projection images is often reduced during the experiment, leading to degradation of the reconstruction quality. Over the last decade, different reconstruction techniques have been developed that yield superior results for such limited tilt series. ${ }^{[14-17]}$ These reconstruction techniques typically try to exploit prior knowledge about the reconstructed object. The discrete algebraic reconstruction technique uses prior knowledge about the gray levels during the reconstruction whereas compressive sensing based reconstruction techniques exploit the sparsity of the object in a given basis. ${ }^{[15-17]}$ However, these methods are not always applicable, due to the lack of prior knowledge. An alternative method to improve the reconstruction quality is by increasing the sampling rate through sinogram interpolation. ${ }^{[18-22]}$ For X-ray computed tomography, sinogram interpolation methods were already successfully applied to minimize streak and ring artifacts. ${ }^{[19]}$ In this paper, we will start by showing the benefits of sinogram interpolation for electron tomography using phantom objects and then this method will be applied for different experimental TEM datasets. 

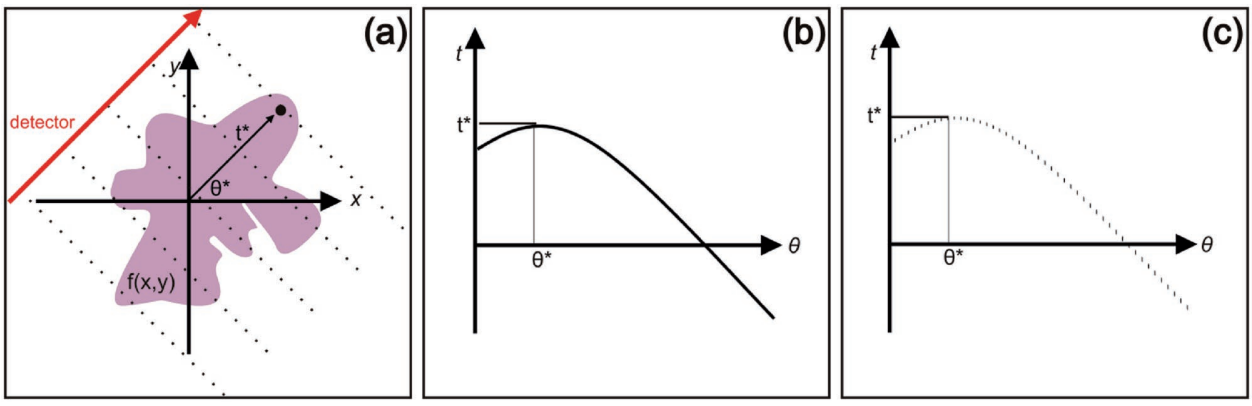

Figure 1. a) 2D object of which a point source corresponds to a sine wave in Radon space b). In case less projections are available, the sine wave will be sparse c).

\section{Methodology}

\subsection{Radon Space and Sinogram}

In order to simplify the description of the methodology, we consider a $2 \mathrm{D}$ object represented by the function $\mathrm{f}(\mathrm{x}, \mathrm{y})$ which is shown in Figure 1a. Then its Radon transform $p(t, \theta)$ is given by ${ }^{[23]}$

$p(t, \theta)=\int_{-\infty}^{\infty} \int_{-\infty}^{\infty} f(x, \gamma) \delta(t-x \cos \theta-\gamma \sin \theta) \mathrm{d} x \mathrm{~d} \gamma$

where $t$ and $\theta$ are the distance and the projection angle of the center of rotation, respectively.

For a single point source $f(x, y)=\delta\left(x-x^{*}, y-y^{*}\right)$, the Radon transform is given by

$$
p(t, \theta)=\delta\left(t-x^{*} \cos \theta-y^{*} \sin \theta\right)
$$

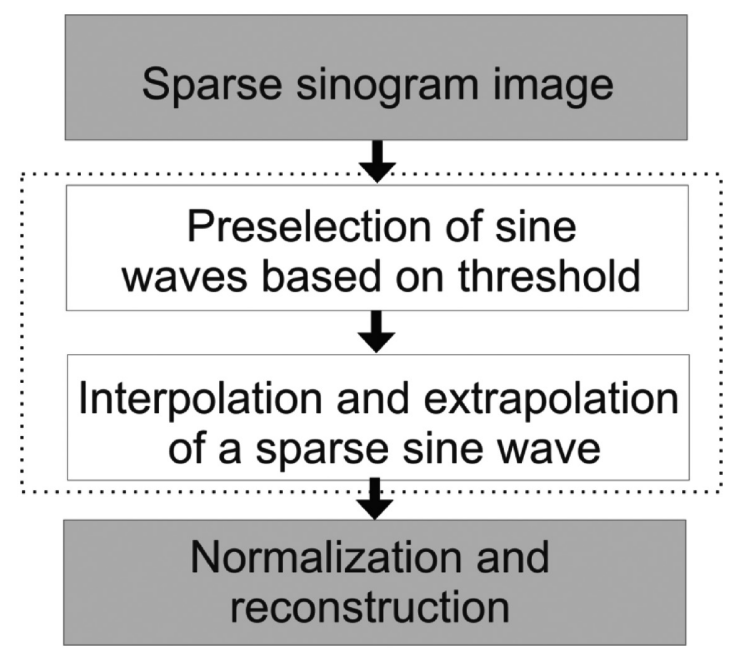

Figure 2. Flowchart of the sine wave preselection and interpolation procedure. The sine waves that are part of a recorded object are first identified based on a predefined threshold. Next, interpolation of the missing sinogram columns and the intensity values is carried out. After summing up all the interpolated sine waves and normalization, the resulting sinogram is used as input for the tomographic reconstruction algorithm. The sinogram interpolation procedure is applied slice by slice on a 3D datacube.
The Radon transform of an object is often referred as a sinogram because the transform of an off-center point source in real space corresponds to a sine wave in Radon space. Indeed, when a point object in real space $(x, y)$ is tilted around an axis, a sine wave $(\theta, t)$ is generated in the sinogram (see Figure $1 \mathrm{~b}$ ). Since an object consists of many point objects, the Radon transform of an object consists of overlapping sine waves with different amplitudes and phases. By acquiring a higher number of projection images, a higher sampling of the sinogram is achieved. If the object is imaged along all projections, then the inverse Radon transform yields the original object. In experiments, we can only take a limited number of projection images (i.e., sparse sinogram Figure 1c) which yields a reduction of the reconstruction quality of the object. ${ }^{[24]}$

A reconstruction of an object can be obtained by filtering each sinogram column with a ramp filter to correct for uneven sampling of the spatial frequencies ${ }^{[1,25]}$ and then backprojecting it along the projection angle. To overcome the problem of limited number of projections, we present a sinogram interpolation method. The methodology of our approach is schematically illustrated in Figure 2. The method is composed of three main stages: (i) preselection of the sine waves based on a threshold, (ii) interpolation and/or extrapolation of a sparse sine wave, and (iii) normalization and then reconstruction.

In order to illustrate the main steps of the flowchart diagram, we will use a phantom object consisting of a homogenous sphere embedded in a homogeneous support (Figure 3a). We assume projection data for a tilt range between $\pm 90^{\circ}$ with a tilt interval of $1^{\circ}$. A slice through the $3 \mathrm{D}$ object, taken orthogonal to the tilt axis, is shown in Figure $3 \mathrm{~b}$. The main steps of the flow chart diagram will be explained in the following sections in more detail.

\subsection{Preselection of Single Sine Waves}

From Equation (2), it can be understood that every pixel in the reconstructed slice corresponds to a particular sine wave in the sinogram. Figure $3 \mathrm{c}$ shows the calculated sinogram for the slice in Figure $3 \mathrm{~b}$ under the experimental conditions mentioned above.

The first step of our method consists of creating an empty matrix with a size equal to the size of the $2 \mathrm{D}$ projection images from the tilt series. Next, for each pixel in a slice, sine waves 

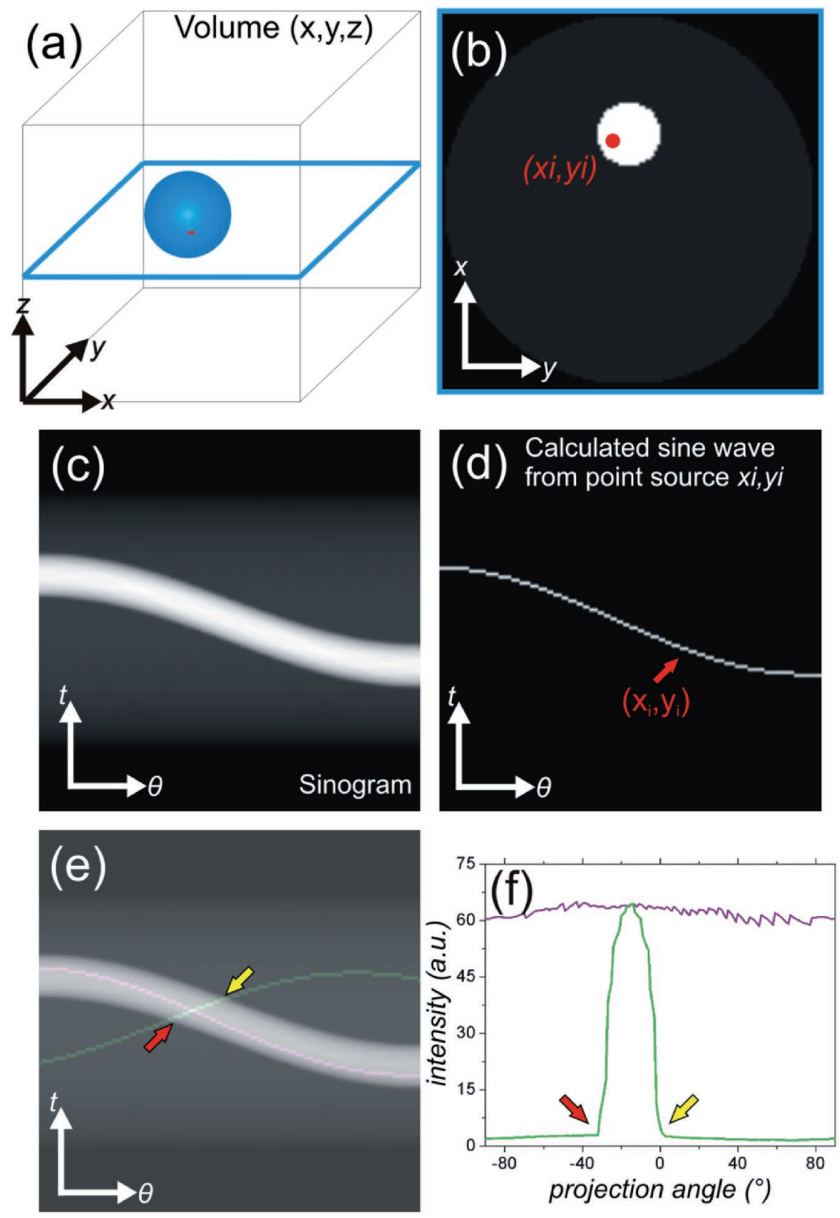

Figure 3. a) A reconstruction volume with a highlighted reconstruction slice. b) A reconstruction slice of the object to be reconstructed, c) the corresponding (recorded) sinogram from the object, d) a single sine wave from a point source $\left(x_{i}, y_{i}\right)$, e) the overlay image of the sine waves with the sinogram, f) the intensities of the sinogram along the two sine curves depicted in (c)

are calculated using Equation (2) hereby using the experimental projection angles $(\theta)$. A representative sine wave is presented in Figure 3d. This sine wave was calculated for the red voxel in Figure 3a and corresponding pixel in Figure 3b. Next, we determine if the sine wave corresponds to a pixel that is possibly part of the object, or if the pixel certainly belongs to the background. The calculated sine wave is therefore superimposed on the experimental sinogram (see Figure 3e) and for every angle $\theta$, the intensity value of the sinogram at the position of the calculated sine wave is plotted (Figure $3 \mathrm{f}$ ).

As an example, two sine waves (depicted in purple and green) are presented in Figure 3e. From Figure 3f, it can be seen that the intensity values extracted based on the purple sine wave are relatively high for all values of $\theta$, whereas the values corresponding to the green sine wave are only significantly different from zero for a given range of $\theta$. From Figure $3 f$, we then conclude that the purple sine wave possibly corresponds to a pixel of the object whereas the green sine wave corresponds to a pixel that certainly belongs to the background. This procedure is repeated for each pixel $(x, y)$ in each slice of the reconstruction.
In general, we assume that pixels may belong to the object if the corresponding sine wave yields intensities above a certain threshold throughout the entire sinogram. This threshold is chosen to be slightly above the intensity value of the homogeneous support. In practice, one can estimate this intensity from a projection image acquired at $0^{\circ}$. The choice of the threshold may be somewhat arbitrary and may give rise to the wrong selection of some points just outside of the contour. However, the relative fraction of those pixels is small in comparison to the total number of internal pixels.

\subsection{Interpolation of a Sparse Sine Wave}

Once a sine wave is considered as a part of the object and the intensity values of the sine wave in each experimental projection is obtained, the next step is to calculate the trajectory of the sine wave for the intermediate (unsampled) angles and to interpolate the intensity of the sine wave through the experimental points. This is performed by applying Equation (2) for the same point source, but with a finer tilt interval. Once the sine wave trajectory is determined, we estimate the intensity value of the sine waves by using a $1 \mathrm{D}$ cubic interpolation method. In principle, the interpolation of the intensity can be performed for every calculated sine wave. However, we prefer to apply intensity interpolation only for the sine waves, which were classified as originating from the investigated object. In this manner, interference of the sine waves from different sources is reduced. Next, the total intensity (corresponding to the sum of all sine waves) for a specific coordinate is divided by the number of sine waves passing through that coordinate. In Figure $4 \mathrm{a}$, we assume that projection images of the object in Figure $3 \mathrm{~b}$ are acquired over a tilt range of $\pm 90^{\circ}$, but now with a tilt interval between successive projections of $30^{\circ}$. Therefore, the sinogram is only based on seven projections. The weighted back projection (WBP) reconstruction from the sparse sinogram is presented in Figure $4 \mathrm{~b}$ and it can be seen that the quality of this reconstruction is poor with the obvious presence of streak artifacts. To minimize such artifacts, we apply the methodology explained above. We first calculate the trajectories of the sine waves for all pixels in every reconstruction slice with a tilt range of $\pm 90^{\circ}$ and an increment of $30^{\circ}$. An example of a preselected and interpolated single sine wave is presented in Figure $4 c, d$, respectively. The final interpolated sinogram is presented in Figure $4 \mathrm{e}$ and the corresponding reconstruction is shown in Figure 4f. We hereby used the classical WBP scheme to judge the improvement of the reconstruction. Every artificially generated column in a sinogram actually represents a projection in real space. Increasing the number of projections by means of sinogram interpolation decreases the artifacts present in the reconstruction. It can be seen that streak artifacts in the reconstruction (Figure $4 \mathrm{f}$ ) are greatly reduced in comparison to Figure 4b.

\subsection{Reduction of Missing Wedge Artifacts}

Typically, a tilt series in an electron tomography experiment covers a range of $\approx \pm 70^{\circ}-80^{\circ}$. Projections outside of this range 

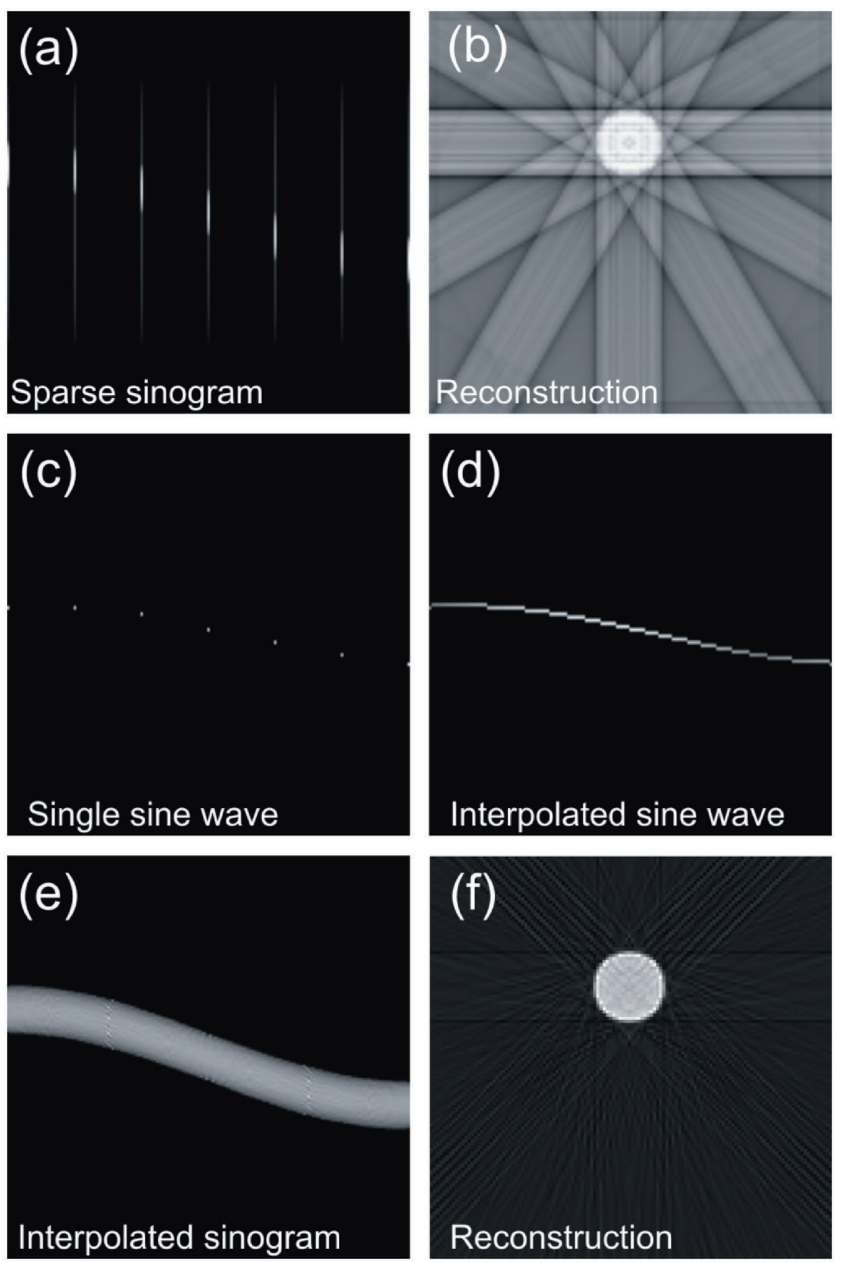

Figure 4. a) A sparse sinogram obtained for a tilt series with a tilt range of $\pm 90^{\circ}$ and tilt increment of $30^{\circ}, b$ ) the reconstruction from the sparse sinogram, c) individual sine wave selected from the sparse sine wave, d) the sine wave in (c) after interpolation, e) the interpolated sinogram, and $f$ ) the reconstruction from the interpolated sinogram.

are difficult to be recorded due to the shadowing from the single tilt holder or the TEM grid. This results in missing information in a sinogram, and as a consequence streak artifacts or an elongation of the object is present in the final reconstruction. Here, we extrapolate the missing wedge intensity of the sine wave by exploiting the known symmetry properties of the sinogram which is based on the fact that a projection from top to bottom is the mirror image of the bottom to top image. ${ }^{[26]}$ In Figure 5a, the sinogram based on projections obtained from the object in Figure $3 \mathrm{~b}$ over a range of $\pm 90^{\circ}$ is shown. The gray rectangles in this image indicate the information that will be lost when only a range of $\pm 75^{\circ}$ is covered. The sinogram for this range is also illustrated in Figure $5 \mathrm{~b}$.

To estimate the intensity values of the sine waves, a slightly different procedure is used in comparison to the methodology explained above. First, the sinogram is divided into two parts at $0^{\circ}$ (indicated by a line in Figure $5 \mathrm{~b}$ ). Next, the angular order is reversed in both parts, as illustrated in Figure 5c. To overcome the mismatch, which is now present in the middle of the modified sinogram, one part of the sinogram (in this case part 2) is flipped with respect to the $x$-axis (see Figure 5d) yielding a sinusoid with a different trajectory. The intensity values can now be extrapolated based on the intensities in the first and last acquired projection, corresponding to the projection at $-75^{\circ}$ and a mirrored projection of $+75^{\circ}$. Next, a 1D interpolation was again employed to estimate the intensities in the missing wedge (see Figure 5e). Next, the modification on the sinogram is reversed as illustrated in Figure 5f, which shows good agreement with Figure 5a (full tilt range). The reconstruction based on WBP using the sinograms from Figure $5 b, f$ are shown in Figure $5 \mathrm{~g}$,h, respectively. To evaluate the results quantitatively, we calculated the elongation of the object before and after interpolation. The reconstructions in Figure $5 \mathrm{~g}, \mathrm{~h}$ are segmented by applying a threshold to obtain binary images. Here, the binary images are referred to as segmented images and the phantom in Figure $3 \mathrm{~b}$ is referred to as our reference.

The elongation is quantified along the vertical and the horizontal direction (Figure 5g,h). After segmenting the reconstructions the length of the object is measured in both directions. The elongation is then calculated by dividing the length of the segmented object by the length of the reference object. In the horizontal direction, no elongation is observed for both reconstructions. However, in the vertical direction, an elongation of $10 \%$ is observed for the reconstruction presented in Figure 5g. After the interpolation procedure (Figure $5 \mathrm{~h}$ ) this number decreases to $3 \%$. It is clear that the streak artifacts and the slight elongation of the object are reduced after the interpolation procedure.

\section{Experimental Results}

In this part, we investigate the applicability of our method using experimental datasets. The method is tested using an Au nanoparticle for which many projections are recorded since the particle is stable under the electron beam. Next, the methodology is applied for a beam sensitive $\mathrm{NaYF}_{4}$ nanoparticle.

\subsection{Qualitative and Quantitative Comparison}

In Figure 6a, a HAADF-STEM image of a Au triangle is presented. A tilt series of similar images was recorded over an angular tilt range of $\pm 76^{\circ}$ with a tilt increment of $1^{\circ}$. In the remainder, this tilt series is referred to as the "full tilt series". By removing some projections from the tilt series, sparser tilt series are generated with an increasing tilt interval of $5^{\circ}, 10^{\circ}$, $15^{\circ}, 20^{\circ}$, and $30^{\circ}$. Next, the sinogram interpolation method is applied and reconstructed using WBP as illustrated in Figure 6.

The visualization of WBP reconstruction from full tilt series is presented in Figure $6 \mathrm{~b}$ and in Figure $6 \mathrm{c}$ a reconstruction based on a sparse tilt series with a tilt increment of $30^{\circ}$ is presented. The corresponding result after sinogram interpolation is presented in Figure 6d. To evaluate the goodness of the reconstruction, we calculate the ratio $(r)$ of the misclassified voxels as follows

$r=\frac{\left|\mathrm{Vol}_{\mathrm{rec}}^{\mathrm{thr}}-\mathrm{Vol}_{\mathrm{ref}}^{\mathrm{thr}}\right|}{\operatorname{sum}\left(\mathrm{Vol}_{\mathrm{ref}}^{\mathrm{thr}}\right)} \times 100$ 

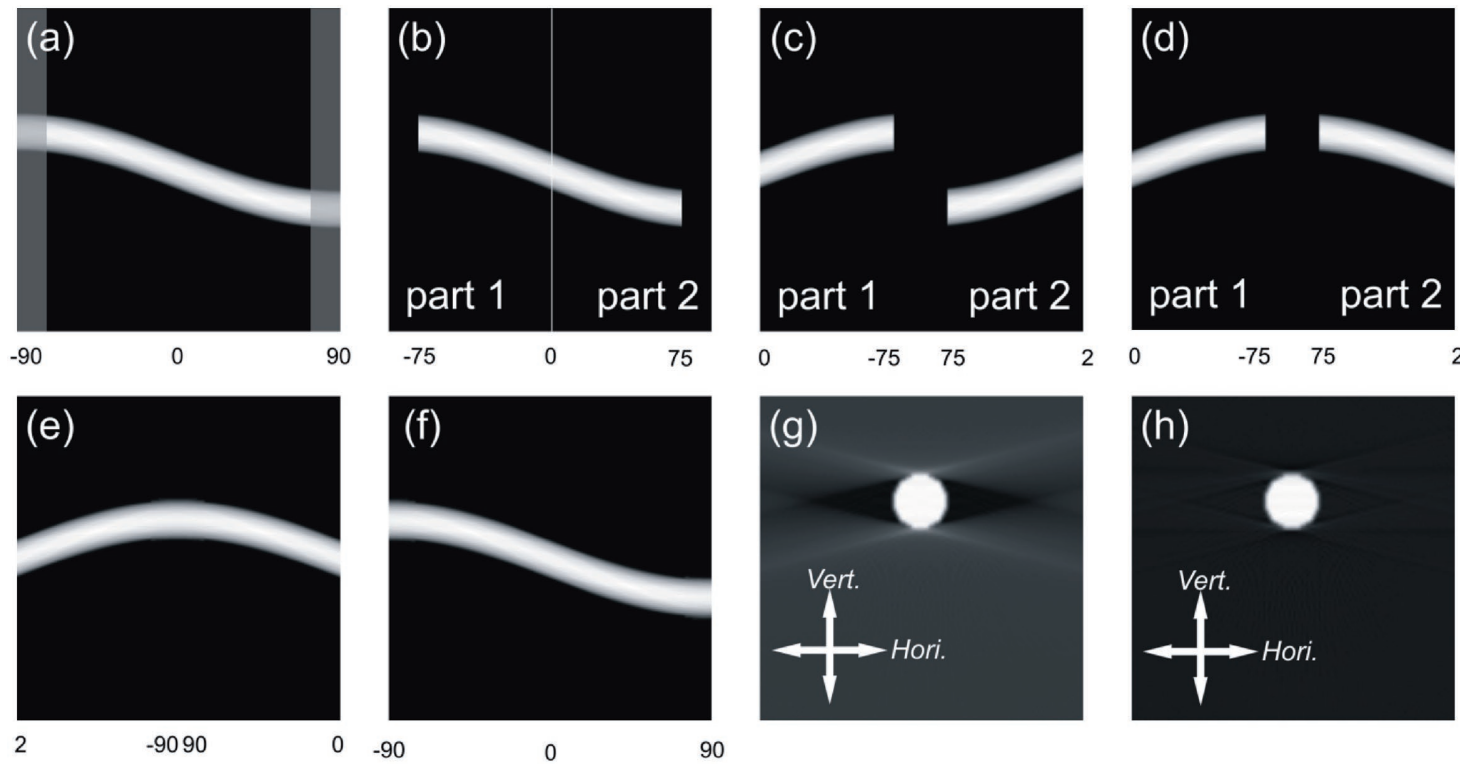

0

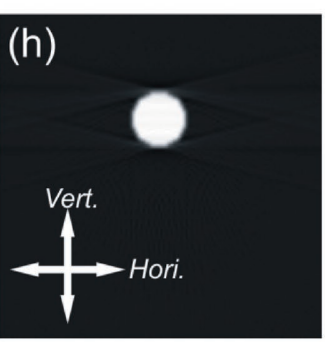

Figure 5. a) The sinogram from an object with projections from $\pm 90^{\circ}, \mathrm{b}$ ) the same sinogram with missing information, c) the sinogram with reordered angles, d) the sinogram with the missing wedge information relocated, e) after interpolation of the missing information, and $f$ ) reversed back sinogram, the reconstructions $\mathrm{g}$ ) with missing wedge and $\mathrm{h}$ ) after interpolation.

$\mathrm{Vol}_{\text {ref }}$ here refers to the segmented volume of the full tilt series and $\mathrm{Vol}_{\text {rec }}$ stands for the segmented volume from sparse or interpolated tilt series. The volume of the segmented object $\operatorname{sum}\left(\mathrm{Vol}_{\text {ref }}\right)$ is equal to the summation of the voxels in $\mathrm{Vol}_{\text {ref }}$ and corresponds to the denominator in Equation (3). A threshold for segmentation of the volumes was determined from the reconstruction with a tilt increment of $1^{\circ}$. Figure 6e shows the misclassified voxels, calculated for reconstructions with increasing tilt increment and clearly indicate a significant error, which can be largely avoided through the method we propose here.

Even though our interpolation method is able to compensate for missing data, the dataset used as an input for WBP is still incomplete. We therefore expect that iterative algorithms such
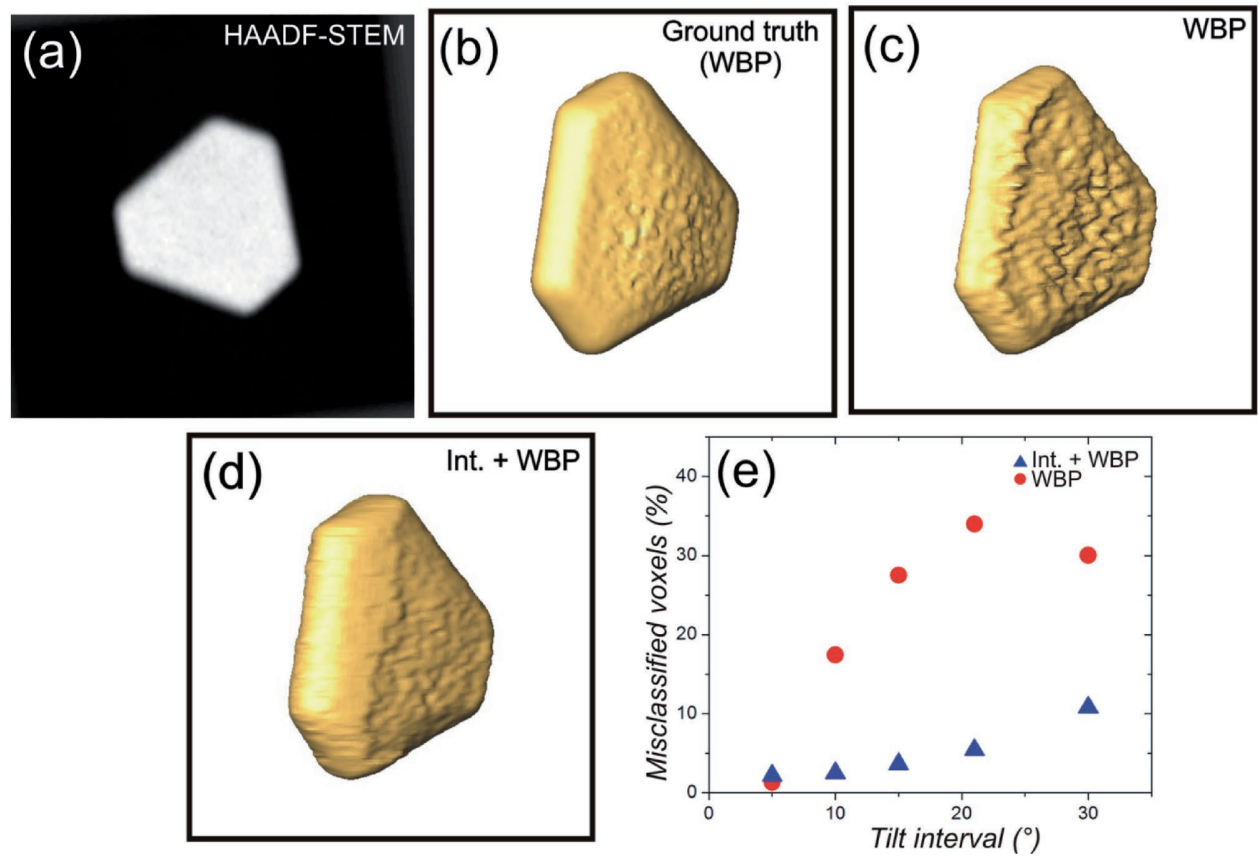

Figure 6. a) HAADF-STEM image from an Au nanotriangle, b) the reconstruction using full tilt series with a projection every $1^{\circ}$, c) the reconstruction using sparse tilt series with a projection every $30^{\circ}$, d) the visualization from the $3 \mathrm{D}$ reconstruction of an interpolated tilt series obtained from the tilt series with a projection every $30^{\circ}$, e) comparison of misclassified voxels between interpolated tilt series and sparse tilt series for different tilt interval. 


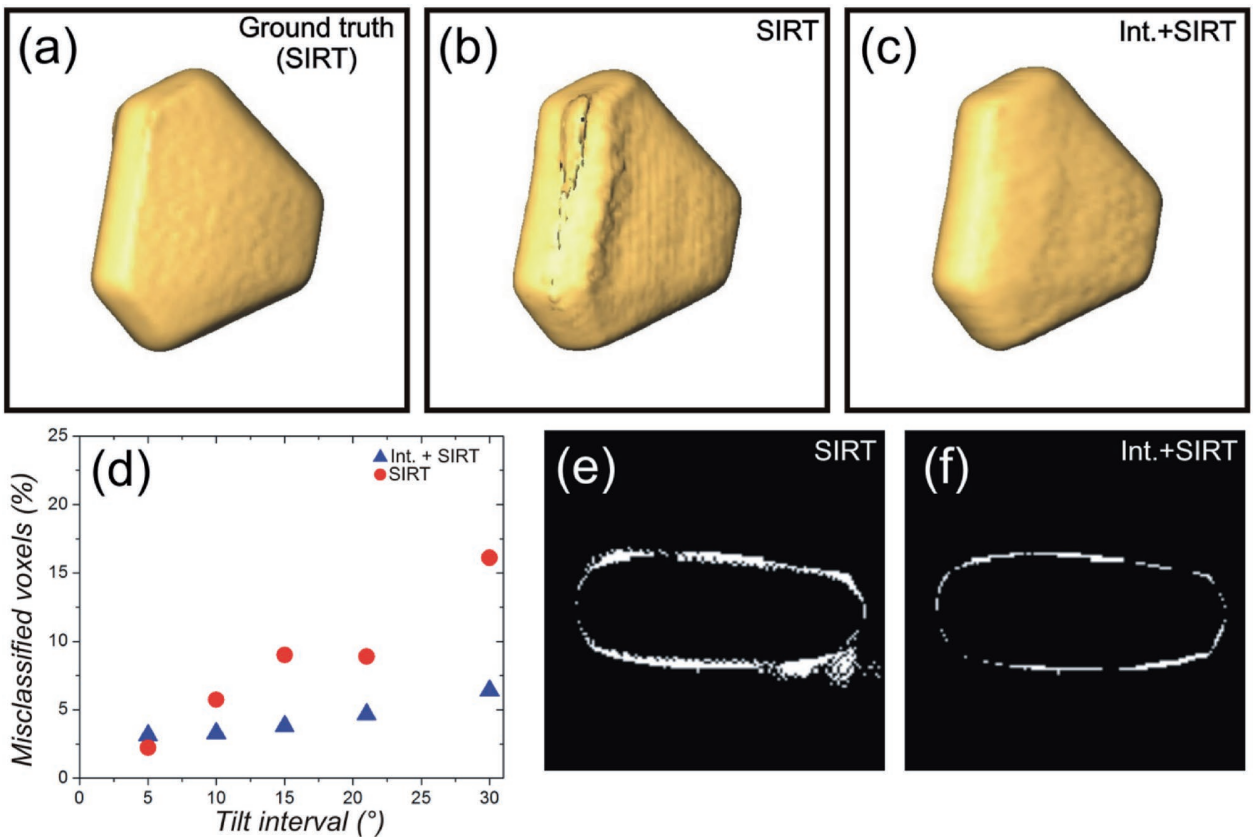

Figure 7. a) The visualization from the 3D reconstruction using full tilt series, b) using sparse tilt series with a projection every $30^{\circ}$, c) and from the interpolated tilt series based on the sparse series, d) the plot for the misclassified voxels as a function of increasing tilt interval. The misclassified voxels comparison between reconstruction from the sparse tilt series e) and the interpolated tilt series $f$ ).

as simultaneous iterative reconstruction technique (SIRT) may still improve the quality of the reconstruction. As a consequence of the sinogram interpolation, new projections are in fact artificially generated. In Video S1 in the Supporting Information, a tilt series of images acquired every $30^{\circ}$ is illustrated. The corresponding tilt series including artificially generated projections is presented in Video S2 in the Supporting Information. These images can also be used as an input for a SIRT. The 3D visualization of the SIRT reconstruction from the full tilt series is presented in Figure 7a. Visualizations of 3D SIRT reconstructions from the sparse tilt series and the tilt series after interpolation are shown in Figure 7b,c, respectively. It is clear that the SIRT reconstruction from the sparse tilt series suffers from severe artifacts at the facets of the nanotriangle. The results based on the tilt series after interpolation (Figure 7c) clearly shows an improved quality in comparison to Figure $7 \mathrm{~b}$ and the morphology of the nanoparticle is in good agreement with the SIRT reconstruction from the full tilt series. Furthermore, the quality of the SIRT reconstruction is improved in comparison to the WBP reconstruction using the same interpolated dataset (Figure 6d).

To evaluate the reconstructions quantitatively, the number of misclassified voxels was calculated for reconstructions with different tilt interval (Figure 7d). The error equals $10 \%$ for a reconstruction based on a series with tilt increment of $15^{\circ}$ and increases up to $18 \%$ in case of a tilt increment of $30^{\circ}$. However, the percentage of misclassified voxels for the reconstructions using the interpolated tilt series never exceeds $6 \%$. The misclassified voxels in the reconstruction based on only six projections are presented in Figure 7e whereas Figure 7f shows the misclassified voxels for the reconstruction using the interpolated tilt series. For both reconstructions, a slight overestimation of the volume is observed, albeit limited for Figure $7 f$.

\section{2. $\mathrm{NaYF}_{4}$ Hexagonal Plates}

To illustrate the potential of our approach when investigating beam sensitive samples, $\mathrm{NaYF}_{4}$ hexagonal platelets were investigated. These nanoparticles can act as efficient luminescent hosts in applications such as biological diagnosis and solar cells. ${ }^{[27,28]}$ In order to optimize and tune their properties, a complete $3 \mathrm{D}$ characterization of their morphology is required. Figure $8 \mathrm{a}$ shows a HAADF-STEM image of an $\mathrm{NaYF}_{4}$ nanoparticle with a size of $\approx 30 \mathrm{~nm}$ and a thickness of $\approx 20 \mathrm{~nm}$. First, a conventional HAADF-STEM tomography experiment was envisioned and a tilt series was acquired from $-72^{\circ}$ to $72^{\circ}$ with $2^{\circ}$ intervals and an acceleration voltage of $200 \mathrm{kV}$. However, when comparing a HAADF-STEM projection acquired at the same tilt angle before (Figure 8a) and after acquisition of the entire tilt series (Figure 8b), sample degradation due to the electron beam is observed. The beam damage mainly induces holes in the 3D structure and the morphology also changes. Therefore, the tilt series cannot be used as an input for 3D reconstruction.

Next, a tilt series of only seven projection images was acquired from another nanoparticle $\left(-74^{\circ}\right.$ to $70^{\circ}$ with a tilt increment of $24^{\circ}$ ) and used as an input for sinogram interpolation. The interpolated tilt series was reconstructed using a WBP and an SIRT reconstruction algorithm. An isosurface rendering of the $3 \mathrm{D}$ reconstruction is presented in the Figure $8 \mathrm{c}, \mathrm{d}$, clearly showing the hexagonal shape of the nanoparticle.

\section{Discussion}

Although the proposed sinogram interpolation method does not explicitly incorporate prior knowledge about the object, 

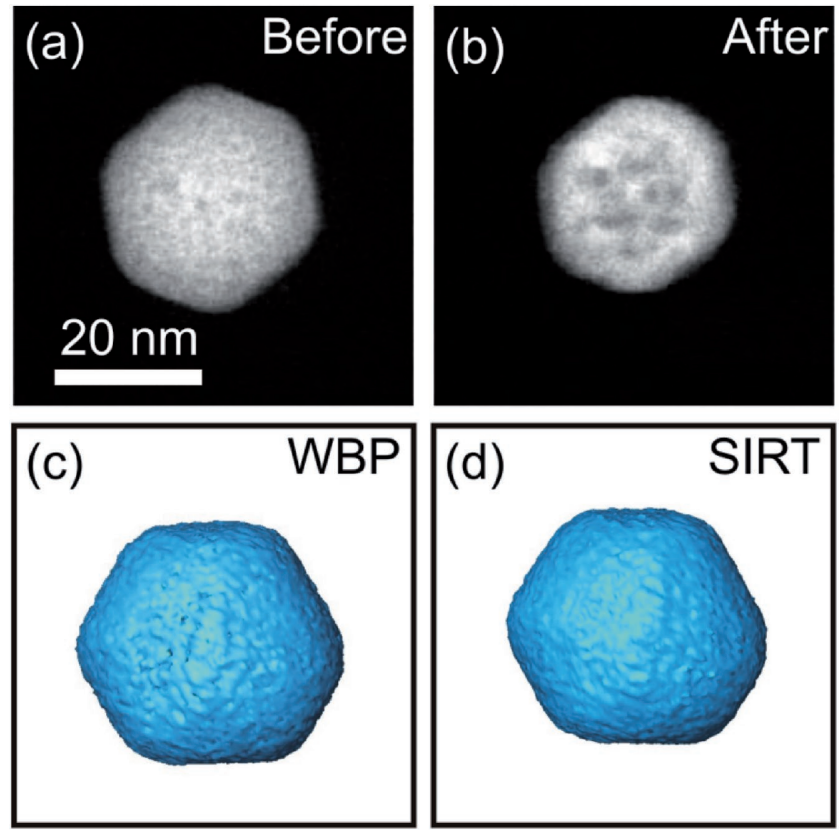

Figure 8. a) HAADF-STEM image from an $\mathrm{NaYF}_{4}$ before the electron tomography experiment and b) after the electron tomography experiment, c) the visualization of the reconstruction using only seven projections in combination with sinogram interpolation, d) the visualization of the reconstruction using SIRT algorithm in combination with sinogram interpolation.

the method incorporates such prior knowledge in an implicit way, through the assumptions that are used for the interpolation procedure. The first key assumption is that image pixels for which the corresponding sine wave falls below the minimal threshold for some data points in the measured sinogram do not belong to the object. This assumption may be violated when imaging thin structures, which have only a small contribution to the sinogram intensity. A second key assumption used in our approach is to consider the sinogram as smooth and continuous, such that interpolation of the measured data points provides a suitable estimate of the intermediate points. This assumption is valid for structures that vary slowly from one projection to the next (i.e., have limited angular dependence), while structures for which the projections varies wildly from one angle to the next will be less suitable. Finally, we consider here particle structures that can be observed in isolation from possible other, surrounding particles. This property is important to enable effective selection of the sinogram waves that contribute to the particle under investigation.

In general, we can say that for individual convex particles with a diameter that is substantially larger than the pixel size, all key assumptions will often be well satisfied. As evidenced by our experimental results, the proposed method can lead to a strong improvement in image quality for such particles.

\section{Conclusions}

In this study, we propose an approach to increase the reconstruction quality of sparse sampled tilt series by means of sinogram interpolation. The applicability of the method was demonstrated using a tilt series of a Au nanotriangle. Conventional reconstructions based on tilt series with different sparsity were compared with reconstructions after the interpolation procedure. Both a qualitative and a quantitative comparison show that the method we propose here results in a significant improvement of the reconstruction. The interpolation method was furthermore applied to a $\mathrm{NaYF}_{4}$ nanoparticle where electron beam damage prevents the acquisition of a tilt series with a relatively small tilt increment. A tilt series of only seven projection images was acquired and used as an input for the proposed interpolation method and a SIRT reconstruction. In this manner, we could determine the morphology of the sample. We conclude that our methodology is of great interest to reconstruct individual convex particles with a diameter that is substantially larger than the pixel size of the projection images.

\section{Supporting Information}

Supporting Information is available from the Wiley Online Library or from the author.

\section{Acknowledgements}

K.S. and S.B. acknowledge support from the Fund for Scientific ResearchFlanders (FWO) (G019014N and G021814N). S.B. acknowledges financial support from European Research Council (ERC Starting Grant \#335078-COLOURATOM). Y.Z. acknowledges financial support from the European Union's Horizon 2020 research and innovation programme under the Marie Skłodowska-Curie Grant Agreement No. 665501 through a FWO [PEGASUS] ${ }^{2}$ Marie Skłodowska-Curie fellowship $(12 \mathrm{U} 4917 \mathrm{~N})$. The authors would like to thank Prof. Luis Liz-Marzán for provision of the samples.

\section{Conflict of Interest}

The authors declare no conflict of interest.

\section{Keywords}

artifact reduction, electron tomography, sinogram interpolation

Received: August 2, 2017 Revised: September 7, 2017 Published online:

[1] P. A. Midgley, M. Weyland, Ultramicroscopy 2003, 96, 413.

[2] P. A. Midgley, R. E. Dunin-Borkowski, Nat. Mater. 2009, 8, 271.

[3] B. Goris, S. Bals, W. Van den Broek, E. Carbó-Argibay, S. Gómez-Graña, L. M. Liz-Marzán, G. Van Tendeloo, Nat. Mater. 2012, 11, 930

[4] A. J. Koster, U. Ziese, A. J. Verkleij, A. H. Janssen, K. P. de Jong, J. Phys. Chem. B 2000, 104, 9368.

[5] S. Bals, G. Van Tendeloo, C. Kisielowski, Adv. Mater. 2006, 18, 892.

[6] A. Koster, R. Grimm, D. Typke, J. Struct Biol. 1997, 120, 276.

[7] J. Frank, Electron Tomography, Springer, New York 2006. 
[8] T. Altantzis, B. Goris, A. Sánchez-Iglesias, M. Grzelczak, L. M. Liz-Marzán, S. Bals, Part. Part. Syst. Charact. 2013, 30,84 .

[9] K. Sentosun, M. N. Sanz Ortiz, K. J. Batenburg, L. M. Liz-Marzán, S. Bals, Part. Part. Syst. Charact. 2015, 32, 1063.

[10] A. Genc, L. Kovarik, M. Gu, H. Cheng, P. Plachinda, L. Pullan, B. Freitag, C. Wang, Ultramicroscopy 2013, 131, 24.

[11] D. Zanaga, F. Bleichrodt, T. Altantzis, N. Winckelmans, W. J. Palenstijn, J. Sijbers, B. de Nijs, M. A. van Huis, A. Sánchez-Iglesias, L. M. Liz-Marzán, A. van Blaaderen, K. Joost Batenburg, S. Bals, G. Van Tendeloo, Nanoscale 2016, 8, 292.

[12] L. Yedra, A. Eljarrat, R. Arenal, E. Pellicer, M. Cabo, A. López-Ortega, M. Estrader, J. Sort, M. D. Baró, S. Estradé, F. Peiró, Ultramicroscopy 2012, 122, 12.

[13] M. Pfannmöller, H. Heidari, L. Nanson, O. R. Lozman, M. Chrapa, T. Offermans, G. Nisato, S. Bals, Nano Lett. 2015, 15, 6634.

[14] P. Gilbert, J. Theor. Biol. 1972, 36, 105.

[15] B. Goris, W. Van den Broek, K. J. Batenburg, H. Heidari Mezerji, S. Bals, Ultramicroscopy 2012, 113, 120

[16] R. Leary, Z. Saghi, P. A. Midgley, D. J. Holland, Ultramicroscopy 2013, 131, 70
[17] K. J. Batenburg, S. Bals, J. Sijbers, C. Kübel, P. A. Midgley, J. C. Hernandez, U. Kaiser, E. R. Encina, E. A. Coronado, G. Van Tendeloo, Ultramicroscopy 2009, 109, 730.

[18] R. Chityala, K. R. Hoffmann, S. Rudin, D. R. Bednarek, Proc. SPIEInt. Soc. Opt. Eng. 2005, 5747, 2110.

[19] Y. Li, Y. Chen, Y. Hu, A. Oukili, L. Luo, W. Chen, C. Toumoulin, J. Opt. Soc. Am. A 2012, 29, 153

[20] E. P. A. Constantino, K. B. Ozanyan, Meas. Sci. Technol. 2008, 19, 94015.

[21] M. Kalke, S. Siltanen, Appl. Math. 2014, 5, 423.

[22] C. E. Widodo, K. Kusminarto, G. B. Suparta, Int. J. Eng. Technol. 2011, 11, 5 .

[23] P. Toft, J. A. Sorensen, Kgs. Lyngby, Technical University of Denmark, Denmark 1996, p. 25.

[24] M. Cao, H. B. Zhang, Y. Lu, R. Nishi, A. Takaoka, J. Microsc. 2010, 239, 66.

[25] S. Bals, B. Goris, T. Altantzis, H. Heidari, S. Van Aert, G. Van Tendeloo, C. R. Phys. 2014, 15, 140.

[26] S. R. Deans, The Transforms and Applications Handbook (Eds: A. D. Poularikas), CRC Press, Boca Raton, FL 2010, Ch. 8.

[27] G. Wang, Q. Peng, Y. Li, Acc. Chem. Res. 2011, 44, 322.

[28] J. Zhou, Z. Liu, F. Li, Chem. Soc. Rev. 2012, 41, 1323. 